\title{
Impacto da degradação sobre o estoque total de carbono de florestas ripárias na Amazônia Oriental, Brasil
}

\author{
Marcelo L. C. ZELARAYÁN ${ }^{1}$, Danielle CELENTANO², Elivaldo C. OLIVEIRA³, Stefanía P. TRIANA ${ }^{1}$, \\ Danilo N. SODRÉ ${ }^{1}$, Karold Henry M. MUCHAVISOY ${ }^{1}$, Guillaume X. ROUSSEAU ${ }^{1 *}$ \\ 1 Universidade Estadual do Maranhão (UEMA), Programa de pós-graduação em Agroecologia, Campus Universitário Paulo VI, s/n, Tirirical, 65054-970, São Luís/MA, Brasil. \\ 2 Universidade Federal do Maranhão (UFMA), Programa de pós-graduação da Rede de Biodiversidade e Biotecnologia da Amazônia Legal (BIONORTE), Campus Universitário do \\ Bacanga, Av. dos Portugueses, 65000-000, São Luís/MA, Brasil. \\ 3 Universidade Federal do Maranhão (UFMA), Programa de pós-graduação em Biodiversidade e Conservação, Campus do Bacanga, Av. dos Portugueses, 65000-000, São Luís/ \\ MA, Brasil. \\ *Autor correspondente: guilirous@yahoo.ca
}

\section{RESUMO}

As florestas ripárias provêem serviços ecossistêmicos essenciais à humanidade, mas estão sendo degradadas em um ritmo acelerado na Amazônia. Esse estudo teve como objetivo quantificar os estoques de carbono acima e abaixo do solo de florestas ripárias com quatro níveis de degradação (muito alta, alta, média e baixa) em duas microbacias (Rio Pepital e Rio Grande) em Alcântara (Maranhão), na Amazônia Oriental. Foram instaladas 24 parcelas permanentes de $1.000 \mathrm{~m}^{2}$, onde a vegetação (árvores, arbustos, herbáceas, lianas e palmeiras), a necromasa (serapilheira, árvores mortas, galhos) e o solo (0-20 cm e raízes) foram amostrados. A biomassa foi estimada mediante modelos alométricos (vegetação com DAP $>1 \mathrm{~cm}$ e árvores mortas) e de forma destrutiva (herbáceas, serapilheira, galhos finos, raízes). O estoque total de carbono nas florestas ripárias mais conservadas variou entre 88 e $202 \mathrm{Mg}(\mathrm{C})$ ha $^{-1}$. A degradação reduziu significativamente o estoque de carbono em todos os compartimentos (até $97 \%$ na biomassa viva acima do solo, até $91 \%$ de necromassa e até $47 \%$ no solo). Nas áreas conservadas, a biomassa viva acima do solo tem a maior participaçáo no estoque total de carbono $(>70 \%)$, principalmente devido às árvores de grande porte. $\mathrm{O}$ estoque de carbono nos diferentes compartimentos estão fortemente relacionados entre si e também com a abertura do dossel. A incorporação desses resultados em modelos regionais de carbono pode auxiliar na implementação e revisão do Código Florestal Brasileiro, em particular na restauração das florestas ripárias onde a agricultura está consolidada.

PALAVRAS-CHAVE: Maranhão, mata ciliar, conservação florestal, serviços ecossistêmicos, sucessão ecológica.

\section{Impact of degradation on carbon stock of riparian forests in the eastern Amazon, Brazil}

\section{ABSTRACT}

Riparian forests provide essential ecosystem services to humanity, but are being degraded at an accelerated rate in the Amazon. This study aimed to quantify carbon stocks above and below ground in riparian forests with four levels of degradation (very high, high, medium and low) in two watersheds (Pepital Rio and Rio Grande) in Alcântara, Eastern Amazon. Twenty-four permanent plots of $1,000 \mathrm{~m}^{2}$ were installed, and vegetation (trees, shrubs, herbs, lianas and palms), necromass (litter, dead trees, branches) and soil (0-20 cm and roots) were sampled. The biomass was estimated using allometric models (vegetation with $\mathrm{dbh}>1 \mathrm{~cm}$ and dead trees) and destructively (herbaceous, litterfall, branches, roots). The total carbon stock in the most conserved riparian forests ranged between 88 and $202 \mathrm{Mg}(\mathrm{C}) \mathrm{ha}^{-1}$. Degradation significantly reduced carbon stock in all compartments (up to $97 \%$ in above-ground biomass, up to $91 \%$ of necromass, and up to $47 \%$ in soils). In conserved areas, the aboveground biomass is the largest compartment of the total carbon stock $(>70 \%)$, mainly due to large trees. The carbon stock in the different compartments are strongly correlated with each other and with the canopy opening. The incorporation of these results in regional carbon models can assist in the implementation and review of the Brazilian Forest Act, particularly in the restoration of riparian forests where agriculture is consolidated.

KEYWORDS: Maranhão, riverine forests, forest conservation, ecosystem services, ecological succession. 


\section{INTRODUÇÃO}

O papel da floresta amazônica na manutenção dos estoques e no fluxo global de carbono é de grande importância (Houghton et al. 2000; Malhi et al. 2008). O desmatamento dessa regiáo é a principal fonte das emissóes brasileiras de dióxido de carbono para a atmosfera (MCT 2013). Atualmente, $20 \%$ da Amazônia já foi desmatada e a porçấo maranhense é a mais destruída (INPE 2014).

O desmatamento e a degradação das florestas ripárias é especialmente preocupante no Estado do Maranhão porque essas florestas protegem a água, recurso que está se tornando escasso. Vastas extensóes de florestas ripárias são destruídas pelo desenvolvimento de cidades, desmatamento para extração de madeira ou pela sua conversão para agricultura devido à fertilidade do solo e acesso facilitado (Junk et al. 2000).

As florestas ripárias funcionam como uma barreira natural que protege os rios da erosão, sedimentaçáo e assoreamento (Rieger et al. 2014), da deposição de agroquímicos (Newbold et al. 2010), além de proporcionar a conservação de peixes, mamíferos, aves e da flora (Jones et al. 1999; Lees e Peres 2008; Bueno et al. 2012). O armazenamento de carbono nas florestas ripárias também é um serviço ambiental de grande relevância para o equilíbrio do clima (Hazlett et al. 2005; Xavier 2009; Rheinhardt et al. 2012).
Apesar da importância dos ecossistemas ripários, ainda existem lacunas de conhecimento sobre o efeito da degradaçáo nos diferentes compartimentos de carbono dessas florestas na Amazônia. A hipótese desse estudo é que a degradação afeta de forma diferenciada os compartimentos do carbono florestal: biomassa arbórea, biomassa arbustiva, biomassa herbácea, necromassa e solo. Dessa forma, este estudo tem como objetivo quantificar os estoques de carbono acima e abaixo do solo de florestas ripárias com diferentes níveis de degradação na Amazônia Oriental.

\section{MATERIAL E MÉTODOS}

\section{Área do estudo}

Esse estudo foi realizado em duas microbacias hidrográficas (Rio Pepital e Rio Grande; Figura 1), parte da grande bacia hidrográfica do Atlântico, localizadas entre a latitude $2^{\circ} 20^{\prime} \mathrm{e}$ $2^{\circ} 23^{\prime} \mathrm{S}$ e a longitude $44^{\circ} 20^{\prime}$ e $44^{\circ} 30^{\prime} \mathrm{O}$, ao norte do Estado do Maranhão, no Município de Alcântara, na Amazônia Oriental. O Rio Pepital tem aproximadamente $11 \mathrm{~km}$ de extensáo, com $17 \mathrm{~km}^{2}$ de área de drenagem e o Rio Grande tem aproximadamente $10 \mathrm{~km}$ desde a sua nascente até a sua foz.

$\mathrm{Na}$ área de estudo vivem remanescentes de quilombos, agricultores e pescadores. O Rio Pepital é o principal
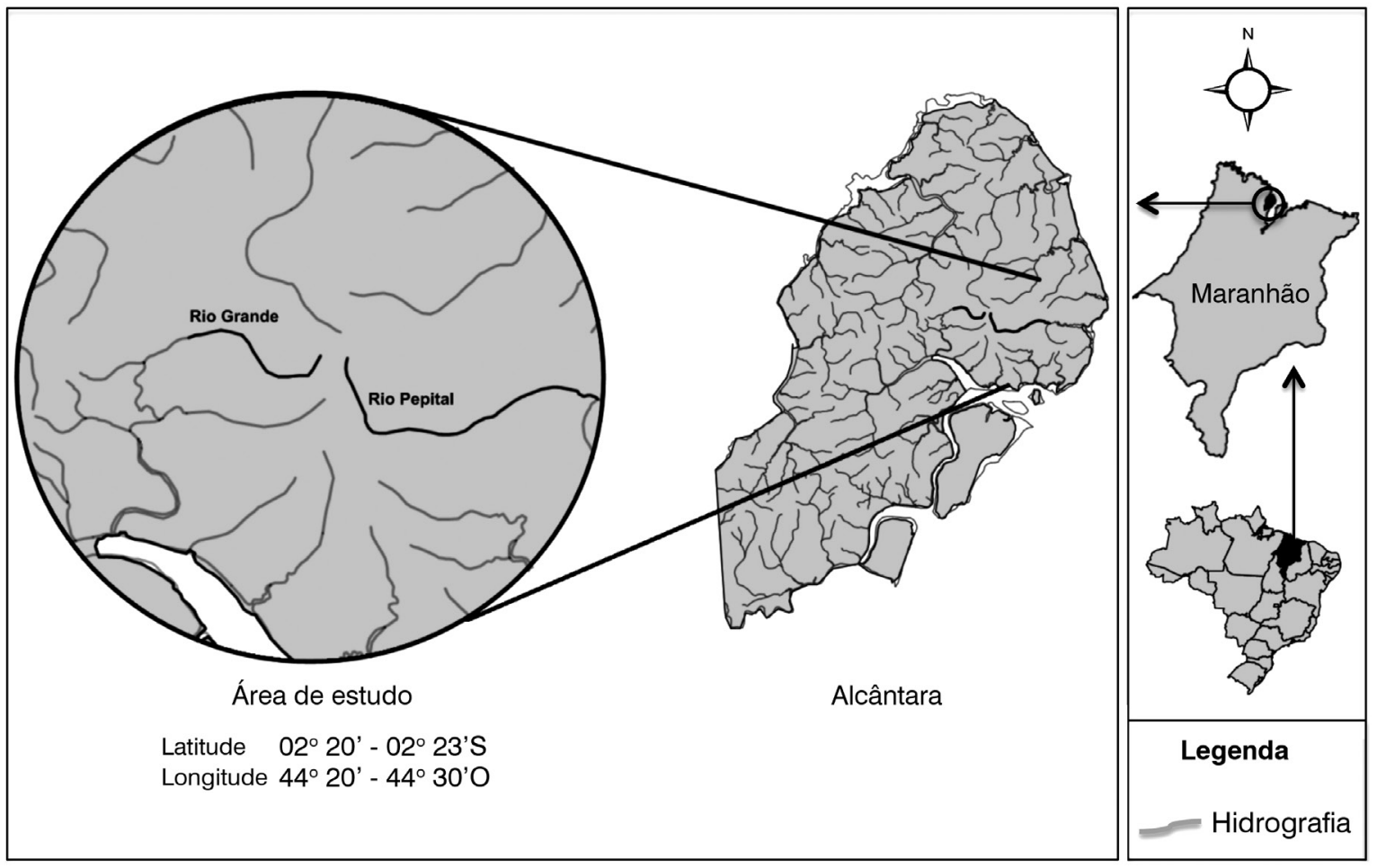

Figura 1. Área de estudo (Rio Grande e Rio Pepital) em Alcântara/ Maranhão, Brasil. 
abastecedor de água do município de Alcântara. O clima na regiáo é quente e úmido com temperatura média de $25^{\circ} \mathrm{C}$ e precipitação anual entre 1.000 e 1.800 mm (Brito e Rego 2001). Florestas secundárias jovens dominam a paisagem, mas ainda existem remanescentes florestais em bom estado de conservação com árvores de grande porte (Celentano et al. 2014). A vegetação dos rios varia de florestas ripárias nas zonas mais altas, florestas alagadas nas partes mais baixas e manguezais na foz. Este estudo foi realizado apenas nas zonas mais altas dos rios, onde só alaga nas enchentes excepcionais.

\section{Desenho experimental}

As florestas ripárias estudadas nos rios Pepital e Grande foram escolhidas após a realização de um diagnóstico rápido das características estruturais da vegetação da zona ripária. Com esse diagnóstico, a vegetação foi classificada em quatro níveis de degradaçáo, a saber: (1) Degradação muito alta - DMA (área aberta, solo aparente ou com algum uso antrópico; ausência de árvores; abertura do dossel > 90\%);
(2) Degradação alta - DA (área em início de sucessão com árvores de até 7 metros de altura; abertura do dossel entre 50 e 90\%); (3) Degradação média - DM (vegetação em estágio avançado de sucessão, presença de árvores com até 15 metros de altura; abertura do dossel < 50\%); e (4) Degradaçáo baixa - DB (floresta secundária com presença de árvores com mais de 15 metros de altura; abertura do dossel < 20\%).

A partir da classificação dos níveis de degradação, foram instaladas 24 parcelas permanentes retangulares de $1.000 \mathrm{~m}^{2}$ ( $50 \times 20 \mathrm{~m}$ ), sendo 12 parcelas em cada local (Rio Pepital e Rio Grande), com três repetiçôes dos quatro níveis de degradaçáo (DMA, DA, DM e DB). As parcelas permanentes foram instaladas paralelamente à margem dos rios com no mínimo 10 metros de distância da área que alaga regularmente. Em cada parcela foram alocadas cinco subparcelas provisórias de $20 \mathrm{~m}^{2}$ (4 x $5 \mathrm{~m}$ ), sendo estas localizadas ao longo da parcela e intercaladas a cada $5 \mathrm{~m}$ (Figura 2). Todas as parcelas estudadas foram georreferenciadas.

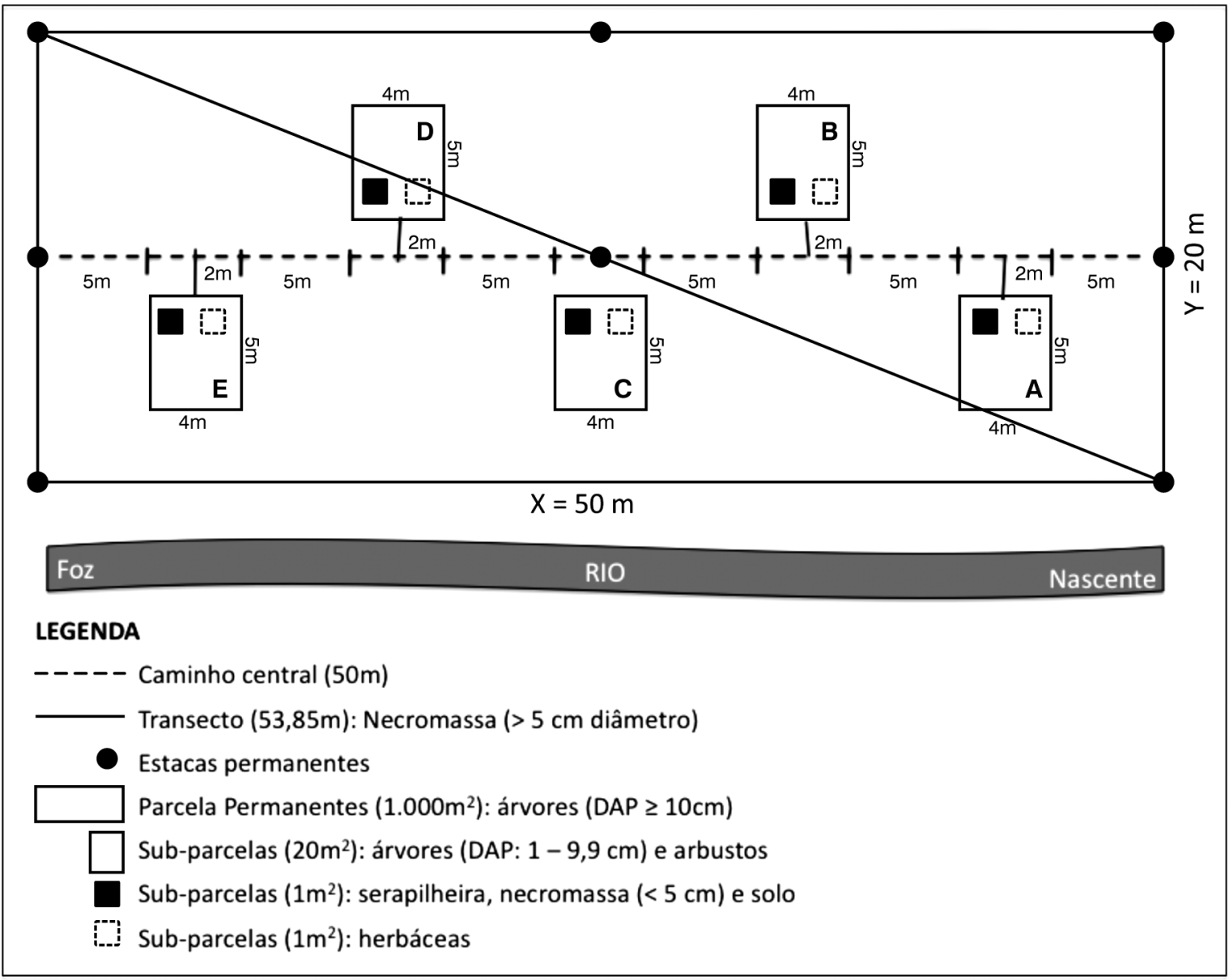

Figura 2. Croqui das parcelas permanentes instaladas para amostragem dos diferentes compartimentos de carbono nas florestas ripárias em Alcântara/ Maranhão, Brasil. 


\section{Abertura do dossel}

A porcentagem de abertura do dossel foi estimada em cada subparcela com o uso de um densiometro florestal convexo. A abertura do dossel de cada parcela correspondeu a média das cinco subparcelas.

\section{Quantificações dos estoques de carbono}

Em cada parcela permanente foi estimado o estoque de carbono da biomassa acima do solo, da necromassa, das raízes e do solo. A metodologia de coleta e análise dos estoques de carbono foi baseada em estudos realizados no trópico úmido (Brown 2002; Chave et al. 2005; Xavier 2009). A coleta de dados no campo foi realizada em outubro e novembro de 2012, com exceção da coleta da vegetação herbáceas que foi realizada em maio de 2013 (estação chuvosa).

\section{Estoques de carbono na biomassa viva acima do solo (BVAS)}

Toda biomassa viva acima do solo (BVAS) com diâmetro na altura do peito (DAP) $>1 \mathrm{~cm}$ foi estimada mediante modelos alométricos mono e multiespecíficos desenvolvidos para a regiáo amazônica e trópico úmido (Tabela 1). Para isso, a vegetação foi estratificada em (1) Biomassa arbórea (árvores e lianas com DAP $>10 \mathrm{~cm}$ e palmeiras com mais de $2 \mathrm{~m}$ de altura do estipe) amostrada em toda a área das parcelas permanentes de $1.000 \mathrm{~m}^{2}$; (2) Biomassa arbustiva (árvores e lianas com DAP entre 1 e $10 \mathrm{~cm}$ e palmeiras com até $2 \mathrm{~m}$ de altura) amostrada nas 5 sub-parcelas de $20 \mathrm{~m}^{2}$ localizadas dentro das parcelas permanentes; (3) Biomassa herbácea (espécies com altura inferior a $1 \mathrm{~m}$ ) amostrada de forma destrutiva em 5 sub-parcelas de $1 \mathrm{~m}^{2}$ (Figura 2).
$\mathrm{O}$ diâmetro das árvores grandes $(\mathrm{DAP}>10 \mathrm{~cm})$ foi mensurado por sutas métricas a altura do peito $(1,30 \mathrm{~m}) \mathrm{e}$ das árvores pequenas com paquímetro na sua base $(30 \mathrm{~cm}$ do solo) e transformado em DAP por meio de equaçôes (Gehring et al. 2008). A altura das árvores foi estimada com um inclinômetro digital e visualmente. A densidade específica da madeira $\left(\mathrm{g} \mathrm{cm}^{-3}\right)$ das espécies arbóreas $(\mathrm{DAP}>10 \mathrm{~cm})$ identificadas taxonomicamente foi consultada em uma base de dados global (Zanne et al. 2009) e, para aquelas espécies náo identificadas, utilizou-se a densidade média $\left(0,583 \mathrm{~g} \mathrm{~cm}^{3}\right)$ de zonas periféricas da região amazônica (Nogueira et al. 2008). A vegetação herbácea foi coletada destrutivamente no campo e seca a $65^{\circ} \mathrm{C}$ até atingir peso constante. Para calcular o estoque de carbono, multiplicou-se a biomassa total pelo fator 0,46 proposto pelo IPCC (2014). A biomassa viva acima do solo (BVAS) foi somada por parcela e extrapolada a $\mathrm{Mg} \mathrm{ha}^{-1}$.

\section{Estoques de carbono na Necromassa (CN)}

O estoque de carbono da necromassa foi quantificado para os seguintes componentes: (1) Serapilheira (folhas, galhos pequenos, flores e frutos $<2 \mathrm{~cm}$ de diâmetro); (2) Galhos finos (galhos entre 2 e $5 \mathrm{~cm}$ de diâmetro); e (3) Madeira morta grossa (árvores mortas em pé e no chão $>5 \mathrm{~cm}$ diâmetro).

A serapilheira e galhos finos foram coletados nas subparcelas de $1 \mathrm{~m}^{2}$ e pesados para determinar a massa fresca. Em seguida, esse material foi homogeneizado e uma amostra de $500 \mathrm{~g}$ foi separada para determinação da umidade. Esse material foi seco a $65^{\circ} \mathrm{C}$ até atingir peso constante e foi calculada a porcentagem de matéria seca. A biomassa das árvores mortas no chão foi estimada pelo método de intercepção plana (Van Wagner 1968); para isso foram percorridos transectos de intercepção de $53,85 \mathrm{~m}$ diagonais às parcelas (Figura 2). Segundo protocolo

Tabela 1. Modelos alométricos utilizados para converter as medidas da vegetação em biomassa viva acima do solo e necromassa. $B=B i o m a s s a ~(k g) ; ~ L n=$ Logarítimo Natural; DAP = Diâmetro a altura do peito (cm); DB = Diâmetro basal (30 acima do nível do solo em cm); di= Densidade específica da madeira $\left(\mathrm{g} \mathrm{cm}^{-3}\right) ; \mathrm{H}=$ Altura $(\mathrm{m})$.

\begin{tabular}{|c|c|c|c|}
\hline Tipo de planta & Tipologia florestal & Equação & Fonte \\
\hline Árvores (DAP > $10 \mathrm{~cm}$ ) & Floresta & $\begin{array}{c}\mathrm{B}=\mathrm{di} * \exp \left(1,499+2,148 \operatorname{Ln}(\mathrm{D})+0,207(\operatorname{Ln}(\mathrm{D}))^{2}-\right. \\
\left.0,0281(\operatorname{Ln}(\mathrm{D}))^{3}\right)\end{array}$ & Chave et al. (2005) \\
\hline Árvores (DAP entre $1-9,9 \mathrm{~cm}$ ) & Floresta secundária & $B=\operatorname{Exp}\{2,4128 \operatorname{Ln}(\mathrm{DAP})-1,9968\}$ & Nelson et al. (1999) \\
\hline Lianas (DB entre $1-13,8 \mathrm{~cm}$ ) & Floresta primária e secundária & $\operatorname{Ln}(B)=-7,114+2,2276 \operatorname{Ln}(D B)$ & Gehring et al. (2004) \\
\hline Palmeiras & Todas & $B=4,5+7,7^{\star} H$ & Frangi e Lugo (1985) \\
\hline Babaçu adulto & Todas & $B=-95,1+49,68(H)$ & Gehring et al. (2011) \\
\hline Árvores mortas em pé & Todas & $\mathrm{B}=0,1184 * \mathrm{DAP}^{2,52 *} 0,04$ & Arevalo (2002) \\
\hline Árvores mortas caídas ( > $5 \mathrm{~cm}$ ) & Todas & $\mathrm{B}=\mathrm{Vol}$ * Densidade & Pearson et al. (2007) \\
\hline Predição DB para DAP em lianas & Floresta primária e secundária & $\mathrm{DAP}=-0,689+(-0,014) * \mathrm{BD}^{2}+1,198 * \mathrm{DB}$ & Gehring et al. (2008) \\
\hline Predição DB para DAP em árvores & Floresta secundária & $\mathrm{DAP}=(-1,038-0,045) * \mathrm{BD}^{2}+1,416 * \mathrm{DB}$ & Gehring et al. (2008) \\
\hline
\end{tabular}


do IPCC (2007), a densidade da madeira das árvores mortas interceptadas foram classificadas em sólidas $\left(0,60 \mathrm{~g} \mathrm{~cm}^{-3}\right)$, intermediárias $\left(0,42 \mathrm{~g} \mathrm{~cm}^{-3}\right)$ e decompostas $\left(0,23 \mathrm{~g} \mathrm{~cm}^{-3}\right)$. Para a determinação da biomassa das árvores mortas em pé aplicou-se o modelo proposto por Arevalo (2002) (Tabela 1).

A concentração de carbono na serapilheira e na madeira fina foi determinada pelo método de combustáo via úmida, onde as amostras foram secas a $65{ }^{\circ} \mathrm{C}$ em uma estufa com circulaçấo forçada de ar até peso constante e depois moídas para análise segundo Walkley e Black (1934). A concentração de carbono da biomassa seca da madeira grossa caída no chão e das árvores mortas em pé foi calculada através da multiplicada pelo fator de 0,46 (IPCC 2014). A necromassa foi somada por parcela e extrapolada a $\mathrm{Mg}(\mathrm{C}) \mathrm{ha}^{-1}$.

\section{Estoque de carbono no solo (COS)}

O estoque de carbono do solo incluiu: (1) o solo; e (2) as raízes finas. Em cada parcela permanente foram retiradas cinco amostras de solo (uma em cada subparcela de $1 \mathrm{~m}^{2}$ ) com cilindros metálicos de volume de $240 \mathrm{~cm}^{3}$ em duas profundidades $(0-10 \mathrm{~cm}$ e $10-20 \mathrm{~cm})$ para calcular a densidade

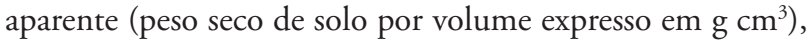
após a secagem do solo a $105^{\circ} \mathrm{C}$ por 48 horas.

O carbono orgânico do solo foi avaliado via combustão úmida (Walkley e Black 1934). Primeiramente, as amostras de solo foram secas ao ar e passadas em peneiras de $2 \mathrm{~mm}$. As cinco subamostras de cada parcela foram homogeneizadas para formar uma amostra composta por profundidade (0-10 e 10-20 cm). O estoque de carbono em $\mathrm{Mg}(\mathrm{C}) \mathrm{ha}^{-1}$ de cada profundidade foi calculado aplicando a seguinte fórmula: $\% \mathrm{C} \times$ (média de densidade aparente da parcela $\mathrm{em} \mathrm{cm}^{-3}$ ) $\times$ (profundidade da camada em $\mathrm{cm}$ ) (Kumar e Nair 2011).

Para a determinação da biomassa de raízes $(<2 \mathrm{~mm})$ foram coletadas 5 amostras de solo por parcela, sendo uma amostra por subparcela (Figura 2) com anel volumétrico de $240 \mathrm{~cm}^{3}$ nas camadas de $0-10$ e 10-20 cm do solo. No laboratório, as raízes foram lavadas com o uso de uma peneira com malha de 2,0 $\mathrm{mm}$ e as raízes vivas foram separadas das mortas. As raízes vivas eram mais claras e maleáveis, enquanto que as mortas eram mais escuras e quebradiças. As raízes vivas foram acondicionadas em sacolas de papel, secas em estufa à $65^{\circ} \mathrm{Ce}$ pesadas para a determinação do peso da massa seca. $\mathrm{O}$ estoque de carbono foi estimado através da multiplicaçáo pelo fator 0,46 (IPCC 2014). As raízes mortas não foram consideradas.

\section{Estoque de carbono total do sistema (CT)}

O estoque total de carbono por unidade de área e nível de degradaçáo foi estimado como $\mathrm{CT}=\mathrm{CBVAS}+\mathrm{CN}+\mathrm{COS}$, expresso em $\mathrm{Mg}(\mathrm{C}) \mathrm{ha}^{-1}$. Onde $\mathrm{CT}=$ Estoque de carbono total, $\mathrm{CBVAS}=$ Estoque de carbono na biomassa viva acima do solo, $\mathrm{CN}=$ Estoque de carbono na necromassa e COS = Estoque de carbono no solo.

\section{Análises estatísticas}

A normalidade dos dados foi avaliada com o teste de Kolmogórov-Smirnov e a homogeneidade da variância com o teste de Cochran, utilizando dados transformados $(\mathrm{Ln}, \mathrm{Ln}+1)$ para atender aos pressupostos da estatística paramétrica. O efeito da degradação das florestas ripárias sobre a abertura do dossel e o estoque de carbono foi avaliado por meio de Análises da Variância (ANOVA) com dois fatores (local, nível de degradação e a interação entre eles) seguida pelo teste de comparação de médias LSD de Fisher $(\alpha=0,05)$. Foram realizadas regressôes lineares para determinar as relaçôes entre os estoques de carbono na biomassa viva acima do solo (BVAS), na necromassa (CN), abaixo do solo (COS) e carbono total. Adicionalmente foram realizadas análises de regressão para determinar a relaçáo da abertura do dossel (\%) com o estoque de carbono nos diferentes compartimentos. Todas as análises foram realizadas com o programa Infostat ${ }^{\circ}(\mathrm{Di}$ Rienzo et al. 2011).

\section{RESULTADOS}

Abertura do dossel - A abertura do dossel das florestas ripárias estudadas apresentou diferença significativa entre os níveis de degradação $(\mathrm{F}=86,61 ; \mathrm{p}<0,0001)$. No entanto não diferiu entre os dois locais estudados ( $\mathrm{p}=0,8598)$ e tampouco houve interaçáo local*nível de degradação $(\mathrm{p}=0,9466)$. Quanto maior o nível de degradação da floresta mais aberto é o dossel (Tabela 2).

Estoque total de carbono - $\mathrm{O}$ estoque total de carbono das florestas ripárias apresentou diferença significativa entre os dois locais estudados $(F=23,20 ; p=0,0002)$, entre os quatro níveis de degradação $(\mathrm{F}=79,20 ; \mathrm{p}<0,0001)$ e também na interação local*nível de degradaçăo $(\mathrm{F}=17,42 ; \mathrm{p}<0,0001)$. As florestas ripárias do Rio Pepital apresentam um estoque total de carbono superior ao Rio Grande e quanto maior o nível de degradação da floresta menor o estoque de carbono nos dois rios (Tabela 2 e Figura 3). Houve efeito significativo da interação entre os fatores (localidade e nível de degradação) porque as áreas mais conservadas (DB) do Rio Grande tem menos carbono total que do Rio Pepital. Comparando as áreas com nível de degradaçấo muito alta (DMA) com as áreas mais conservadas (DB), observa-se uma redução média do carbono total de $135,92 \mathrm{Mg}(\mathrm{C}) \mathrm{ha}^{-1}$, ou seja, uma perda de $87,5 \%$ de carbono.

Nas áreas mais conservadas (DB), o carbono da biomassa viva acima do solo (CBVAS) foi o compartimento que mais contribuiu com o estoque total $(72,4 \%)$, seguido pelo estoque de carbono do solo $(20,4 \%)$ e da necromassa $(7,1 \%)$. No entanto, nas áreas com níveis de degradação alta (DA) e muito alta (DMA) os estoques de carbono abaixo do solo foram os que mais contribuíram para estoque de carbono total, respectivamente 68,5 e $82,3 \%$. Já nas áreas com um nível 
Tabela 2. Abertura do dossel (\%) e estoque de carbono ( $M g$ ha $\left.^{-1}\right)$ dos diferentes compartimentos (biomassa viva acima do solo, necromassa e solo) em florestas ripárias com degradação muito alta (DMA), alta (DA), média (DM) e baixa (DB) em Alcântara/ Maranhão, Brasil. Médias seguidas pelo desvio padrão entre parênteses. Letras distintas indicam diferenças estatísticas entre os níveis de degradação pelo teste LSD Fisher $(p<0,05)$.

\begin{tabular}{|c|c|c|c|c|c|c|c|c|c|c|c|c|}
\hline & \multicolumn{4}{|c|}{ Rio Pepital } & \multicolumn{4}{|c|}{ Rio Grande } & \multicolumn{4}{|c|}{ Média } \\
\hline & DMA & DA & $\mathrm{DM}$ & DB & DMA & $\mathrm{DA}$ & $\mathrm{DM}$ & DB & DMA & DA & $\mathrm{DM}$ & DB \\
\hline $\begin{array}{l}\text { Abertura do dossel } \\
(\%)\end{array}$ & $94,1(10,2)$ & $54,3(25,0)$ & $9,4(1,3)$ & $5,7(1,0)$ & $98,3(1,53)$ & $51,2(14,0)$ & $11,6(7,8)$ & $5,7(0,6)$ & $96,2(6,9) a$ & $52,8(18,2) b$ & $10,5(5,2) c$ & $5,7(0,7) d$ \\
\hline
\end{tabular}

Carbono da Biomassa Viva Acima do Solo (CBVAS)

\begin{tabular}{|c|c|c|c|c|c|c|c|c|c|c|c|c|}
\hline $\begin{array}{l}\text { Árvores } \\
(\mathrm{DAP}>10 \mathrm{~cm})^{*}\end{array}$ & $0,0(0,0) \mathrm{a}$ & $0,0(0,0) a$ & $18,4(7,6) b$ & $149,2(22,2) c$ & $0,0(0,0) a$ & $0,0(0,0) \mathrm{a}$ & $8,6(5,3) a$ & $45,9(23,6) b$ & $0,0(0,0) a$ & $0,00(0,0) \mathrm{a}$ & $13,5(7,9) \mathrm{b}$ & $97,6(60,2) c$ \\
\hline (DAP 1 - 9,9 cm) & $0,2(0,3)$ & $6,0(3,0)$ & $3,5(1,2)$ & $3,1(2,1)$ & $0,4(0,1)$ & $3,1(0,9)$ & $5,7(0,2)$ & $2,7(0,8)$ & $0,3(0,2) a$ & $4,6(2,5) b$ & $4,6(1,4) b$ & $2,9(1,4) b$ \\
\hline Palmeiras & $1,5(2,6)$ & $0,1(0,2)$ & $3,9(3,6)$ & $6,6(5,7)$ & $0,0(0,0)$ & $0,0(0,0)$ & $0,2(0,3)$ & $1,4(0,7)$ & $0,7(1,8)$ & $0,0(0,1)$ & $2,0(3,1)$ & $4,0(4,6)$ \\
\hline Cipós & $0,0(0,0)$ & $0,0(0,0)$ & $0,1(0,1)$ & $0,0(0,0)$ & $0,0(0,0)$ & $0,0(0,0)$ & $0,0(0,0)$ & $0,1(0,1)$ & $0,0(0,0)$ & $0,0(0,0)$ & $0,0(0,0)$ & $0,0(0,1)$ \\
\hline Herbáceas & $1,8(0,9)$ & $1,0(0,6)$ & $0,6(0,3)$ & $0,9(0,3)$ & $1,0(0,8)$ & $0,8(0,2)$ & $0,4(0,1)$ & $0,7(0,3)$ & $1,4(0,9) a$ & $0,9(0,4) a b$ & $0,5(0,3) b$ & $0,8(0,3) a b$ \\
\hline Total CBVAS* & $3,4(2,3) a$ & $7,1(3,7) a b$ & $26,4(10,1) b$ & $159,9(21,5) c$ & $1,5(0,9) a$ & $3,9(1,1) \mathrm{a}$ & $14,9(5,3) a$ & $50,7(23,9) b$ & $2,4(1,9) a$ & $5,5(3,0) a$ & $20,7(9,6) b$ & $105,3(63,2) c$ \\
\hline \multicolumn{13}{|c|}{ Carbono da Necromassa (CN) } \\
\hline $\begin{array}{l}\text { Árvores grandes } \\
\text { caídas no chão }\end{array}$ & $0,7(1,2)$ & $0,0(0,0)$ & $0,3(0,4)$ & $7,8(10,3)$ & $0,0(0,0)$ & $0,0(0,0)$ & $0,8(1,4)$ & $5,1(3,3)$ & $0,3(0,8) a$ & $0,0(0,0) a$ & $0,5(0,9) a$ & $6,5(7,0) b$ \\
\hline Mortas em pé & $0,0(0,0)$ & $0,0(0,0)$ & $0,1(0,0)$ & $0,1(0,1)$ & $0,0(0,0)$ & $0,0(0,0)$ & $0,1(0,1)$ & $0,3(0,4)$ & $0,0(0,0)$ & $0,0(0,0)$ & $0,1(0,1)$ & $0,2(0,3)$ \\
\hline Galhos finos & $0,5(0,8)$ & $0,0(0,0)$ & $0,5(0,2)$ & $1,0(0,4)$ & $0,0(0,0)$ & $0,0(0,0)$ & $0,3(0,2)$ & $0,4(0,2)$ & $0,2(0,5) \mathrm{a}$ & $0,0(0,0) a$ & $0,4(0,2) a b$ & $0,7(0,4) \mathrm{b}$ \\
\hline Serapilheira & $0,4(0,1)$ & $3,0(0,7)$ & $3,1(1,2)$ & $3,5(2,2)$ & $0,4(0,3)$ & $2,2(0,2)$ & $2,8(0,3)$ & $2,6(0,8)$ & $0,4(0,2) a$ & $2,6(0,7) b$ & $3,0(0,8) b$ & $3,1(1,6) b$ \\
\hline Total CN & $1,5(1,8)$ & $3,0(0,7)$ & $3,9(1,7)$ & $12,4(10,0)$ & $0,4(0,3)$ & $2,2(0,2)$ & $4,0(1,8)$ & $8,4(2,5)$ & $0,9(1,3) a$ & $2,6(0,7) b$ & $4,0(1,5) b$ & $10,4(6,9) \mathrm{c}$ \\
\hline \multicolumn{13}{|c|}{ Carbono no Solo (COS) } \\
\hline Raízes $<2 \mathrm{~mm}$ & $1,0(0,6)$ & $1,2(0,5)$ & $1,7(0,2)$ & $2,5(1,0)$ & $0,9(0,3)$ & $1,1(0,4)$ & $1,6(0,3)$ & $1,7(0,5)$ & $0,9(0,4) a$ & $1,2(0,4) a b$ & $1,6(0,2) b$ & $2,1(0,8) \mathrm{C}$ \\
\hline Solo $(0-20 \mathrm{~cm})$ & $13,8(3,8)$ & $15,8(2,8)$ & $18,5(4,3)$ & $27,6(5,5)$ & $15,7(2,9)$ & $17,2(3,2)$ & $21,3(2,3)$ & $27,5(4,2)$ & $14,8(3,2) a$ & $16,5(2,8) a b$ & $19,9(3,4) b c$ & $27,6(4,4) c$ \\
\hline Total COS & $14,8(3,2)$ & $17,0(2,5)$ & $20,2(4,1)$ & $30,2(6,3)$ & $16,6(2,7)$ & $18,3(3,3)$ & $22,8(2,3)$ & $29,2(3,8)$ & $15,7(2,8) \mathrm{a}$ & $17,7(2,7) a b$ & $21,5(3,3) b$ & $29,7(4,7) \mathrm{C}$ \\
\hline & & & & & $18,4(2,3) \mathrm{a}$ & 24,4 (3 & & & 19,0 & 25,8 & & \\
\hline
\end{tabular}

* Apresenta efeito significativo da interação entre o local (Rio Pepital e Rio Grande) e o nível de degradação (DMA, DA, DM e DB).

de degradação médio (DM), a participação dos estoques de carbono da biomassa viva acima do solo e abaixo do solo foram similares, respectivamente 46,6 e $44,8 \%$ do carbono total.

Carbono na biomassa viva acima do solo (CBVAS) - $O$ estoque de carbono na biomassa viva acima do solo (CBVAS) apresentou diferença significativa entre os dois locais estudados $(\mathrm{F}=40,04 ; \mathrm{p}<0,0001)$, entre os quatro níveis de degradação $(\mathrm{F}=95,22$ e $\mathrm{p}<0,0001)$ e em relação à interação local*nível de degradação $(\mathrm{F}=27,35 ; \mathrm{p}<0,0001)$. Assim como o estoque total de carbono, as florestas do Rio Pepital apresentaram um estoque de CBVAS superior ao Rio Grande e quanto maior o nível de degradação da floresta menor o estoque de carbono em ambos rios (Tabela 2 e Figura 3). A interação entre os fatores deve-se ao maior estoque de carbono nas florestas mais conservadas (DB) do Rio Pepital. Comparando o estoque no DMA e DB observa-se que o processo de degradaçáo levou a redução de carbono na CBVAS da ordem de $102,8 \mathrm{Mg}(\mathrm{C})$ $\mathrm{ha}^{-1}$, o que representa uma reduçáo média de $97,7 \%$.

O carbono do componente arbóreo (DAP $>10 \mathrm{~cm}$ ) representa $92,7 \%$ do CBVAS nas áreas mais conservadas (DB), enquanto nas áreas com degradação média (DM) este percentual cai para $65,4 \%$. De fato, nas áreas mais conservadas (DB) o componente arbóreo responde por $67,1 \%$ do estoque total de carbono na floresta e as árvores de grande porte (DAP $>50 \mathrm{~cm}$ ) são responsáveis por $45,9 \%$ desse estoque total. Nas áreas com degradaçáo média (DM), a abundância de árvores 

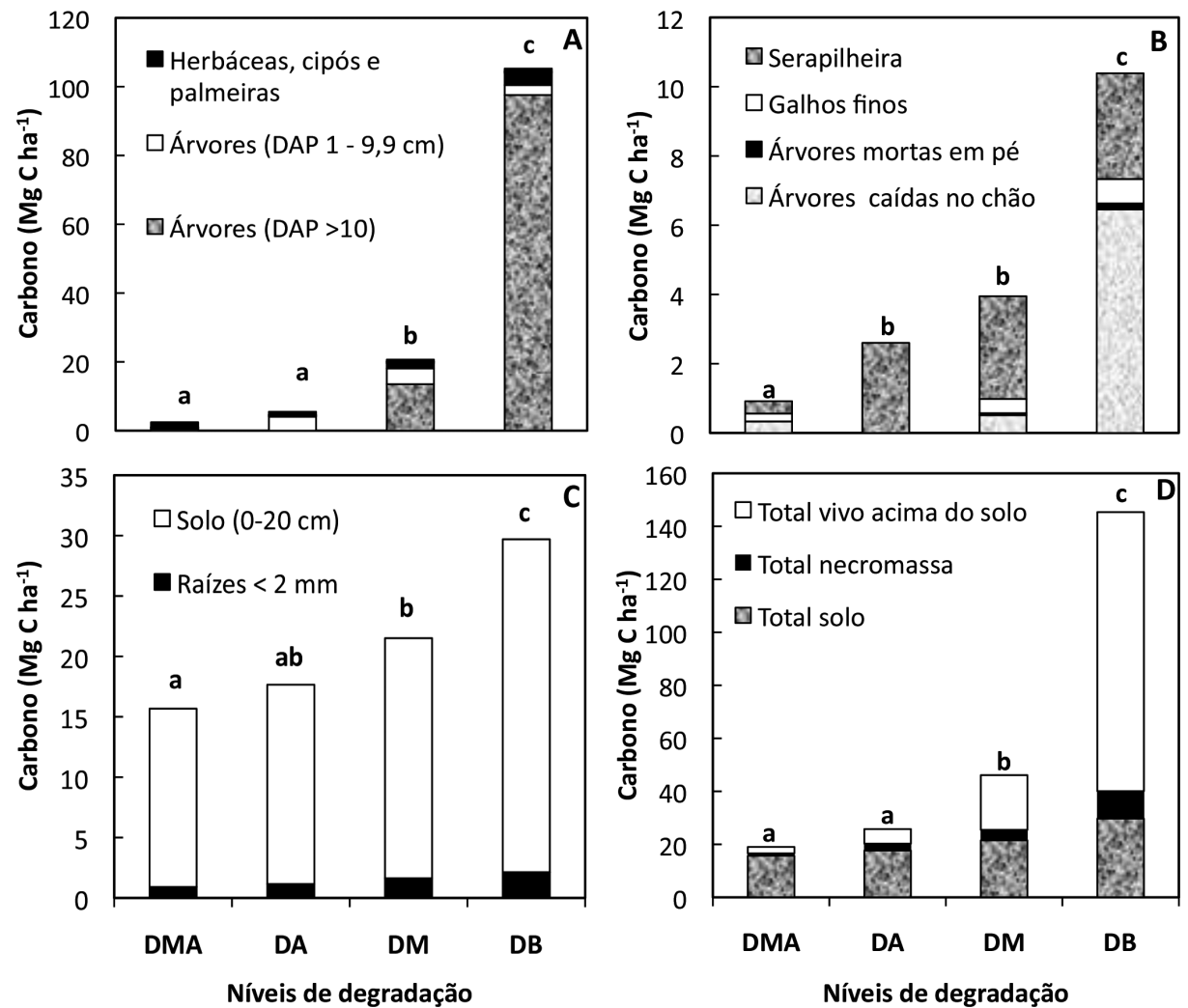

Figura 3. Estoque de carbono ( $\left(\mathrm{gg} \mathrm{ha}^{-1}\right)$ em diferentes compartimentos de florestas ripárias com degradação muito alta (DMA), alta (DA), média (DM) e baixa (DB): (A). Acima do solo; (B). Necromassa; (C). Solo; e (D). Estoque total. Médias obtidas a partir dos dados dos dois locais (Rio Grande e Rio Pepital) amostrados $(n=24)$. Letras distintas indicam diferenças estatísticas entre os níveis de degradação pelo teste LSD Fisher $(p<0,05)$.

com DAP > $50 \mathrm{~cm}$ é zero e são as árvores com menor porte (DAP entre 10 e $29,9 \mathrm{~cm}$ ) as que mais contribuem com o carbono da CBVAS.

Nas áreas com degradação alta (DA) e muito alta (DMA) houve uma eliminaçáo completa das árvores (DAP > 10 $\mathrm{cm}$ ) e o pouco carbono estocado na CBVAS provem das arvoretas e das herbáceas. Quanto maior a degradação, maior a participação do estoque de carbono oriundo do componente herbáceo. O grupo das palmeiras também sofre importante redução com a degradação (82\%), diminuindo conseqüentemente o estoque de carbono desse componente. Os cipós representam a menor contribuição para o estoque de CBVAS e não diferem estatisticamente entre os níveis de degradação (Tabela 2).

Carbono na Necromassa (CN) - O estoque de carbono na necromassa $(\mathrm{CN})$ não apresentou diferença estatística entre os locais estudados, mas sim entre os níveis de degradação $(\mathrm{F}=$ 7,11 e $\mathrm{p}=0,003$ ). As florestas ripárias mais conservadas (DB) apresentam os maiores estoques de carbono na necromassa (Tabela 2). A degradação da floresta leva a uma redução média de carbono na necromassa de $9,5 \mathrm{Mg}(\mathrm{C}) \mathrm{ha}^{-1}$, o que corresponde a uma perda de $91,2 \%$ desse componente. Nas áreas mais conservadas, o carbono das árvores mortas caídas no chão representa $62,2 \%$ do estoque total da necromassa e é seguido pelo carbono da serapilheira com 29,4\% do estoque total.

Carbono abaixo do solo (COS) - Não foram verificadas diferenças significativas para os estoques de carbono abaixo do solo entre os locais estudados e tampouco houve efeito significativo da interação local*degradação $(\mathrm{p}>0,5)$. No entanto, o estoque de carbono apresentou diferença significativa entre os níveis de degradação $(\mathrm{F}=16,87$; $\mathrm{p}<$ $0,0001)$. O estoque de carbono nas raízes e no solo é reduzido significativamente com a degradação da floresta e a diferença desse estoque entre as áreas com degradação baixa (DB) e as áreas muito degradadas (DMA) é de $14,0 \mathrm{Mg}(\mathrm{C}) \mathrm{ha}^{-1}$, representando uma redução de 47,2\%.

Relaçáo entre os compartimentos de carbono - As regressóes lineares (Figuras 4A, 4B e 4C) indicam que há forte relação entre o estoque de carbono na biomassa viva acima do solo (CBVAS) com o estoque de carbono da necromassa $\left(\mathrm{r}^{2}=\right.$ $0,59, \mathrm{p}<0,0001)$, com o estoque de carbono do solo $\left(\mathrm{r}^{2}=0,50\right.$; $\mathrm{p}<0,0001)$ e principalmente com o estoque de carbono total 

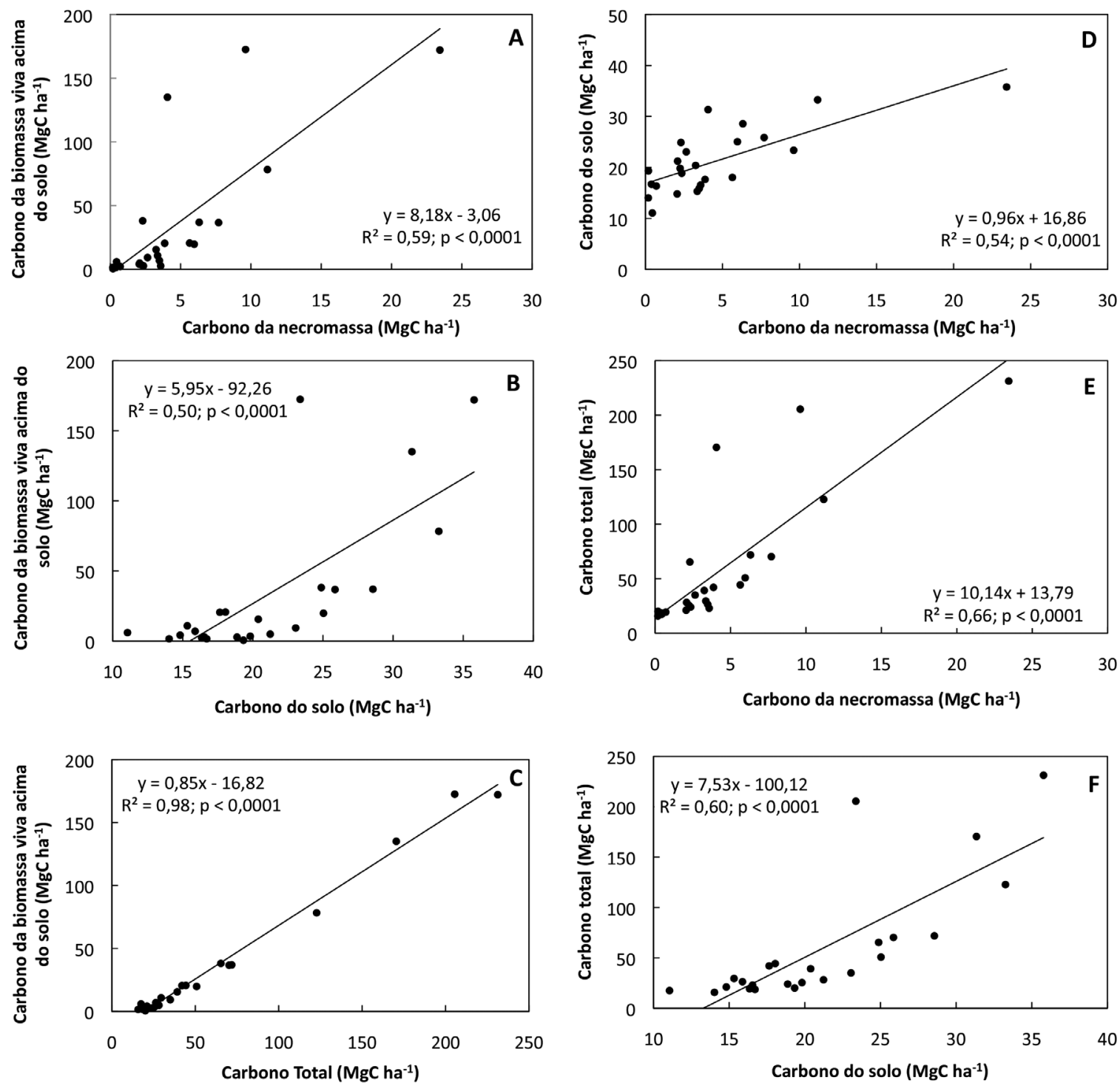

Figura 4. Regressão linear entre os estoques de carbono nos diferentes compartimentos: (A). Biomassa viva acima do solo (CBVAS) e necromassa; (B). CBVAS e solo; (C). CBVAS e estoque total; (D). Necromassa e solo; (E). Necromassa e estoque total; e (F). Solo e estoque total.

das florestas ripárias $\left(\mathrm{r}^{2}=0,98, \mathrm{p}<0,0001\right)$. Da mesma forma, o estoque de carbono da necromassa também mostra forte relação (Figuras 4D e 4E) com o estoque de carbono do solo $\left(\mathrm{r}^{2}\right.$ $=0,54 ; \mathrm{p}<0,0001)$ e o estoque de carbono total $\left(\mathrm{r}^{2}=0,66 ; \mathrm{p}\right.$ $<0,0001)$. Finalmente, o estoque de carbono do solo tem uma relação importante com o estoque total de carbono (Figura 4F) das florestas ripárias estudadas $\left(\mathrm{r}^{2}=0,60 ; \mathrm{p}<0,0001\right)$.

Relação entre a abertura do dossel e os compartimentos do carbono - As análises de regressão indicam que há relação linear negativa entre a abertura do dossel da floresta (\%) e os estoques de carbono $\left(\mathrm{Mg}(\mathrm{C}) \mathrm{ha}^{-1}\right)$ na biomassa viva acima do solo $\left(r^{2}=0,40 ; p<0,0006\right)$, na necromassa $\left(r^{2}=0,35\right.$; $\mathrm{p}<0,0013)$, no solo $\left(\mathrm{r}^{2}=0,55 ; \mathrm{p}<0,0001\right)$ e também com o estoque de carbono total das florestas ripárias $\left(\mathrm{r}^{2}=0,48\right.$; $\mathrm{p}<0,0002)$. Quanto maior a abertura do dossel, menor o estoque de carbono.

\section{DISCUSSÃO}

A avaliação da degradação florestal é uma tarefa difícil devido a alta variabilidade no espaço e no tempo (FAO 2009). Os agentes da degradação antrópica (como madeireiros, agricultores, extrativistas) geralmente deixam áreas altamente degradadas ao lado de outras relativamente bem conservadas. As demais áreas se encontram em situaçōes intermediárias 
variadas, o que dificulta a identificação objetiva de níveis de degradação. Dessa forma, cada degradação se torna única e a construção de um gradiente para fins de investigação deve adaptar-se a cada situaçáo. A escala de degradação construída nesse estudo (degradação muito alta, alta, média e baixa) baseada na abertura do dossel e outros parâmetros estruturais da floresta simples de medir em campo pode servir de base para comparação com estudos futuros.

Esse estudo apresenta a primeira estimativa da diminuição dos estoques de carbono ao longo de um gradiente de degradação em florestas ripárias na Amazônia Oriental, possibilitando a extrapolaçáo para outros ecossistemas ripários da região. Assim será possível ter uma estimativa confiável do potencial de seqüestro de carbono nas áreas a onde a agricultura está consolidada. Nessas áreas, a restauração das florestas ripárias deve ser uma prioridade devido aos benefícios para a proteção dos recursos hídricos.

$\mathrm{Na}$ área estudada, o estoque total de carbono nas florestas mais conservadas variou entre 88 e $202 \mathrm{Mg}(\mathrm{C}) \mathrm{ha}^{1}$, resultado equivalente a estudos realizados em terra firme na regiáo amazônica (Malhi et al. 2006; Xavier 2009). A degradação das florestas ripárias levou a uma redução expressiva no estoque de carbono (até 90\%), principalmente devido à supressão do componente arbóreo. As árvores de grande porte (DAP > $50 \mathrm{~cm}$ ) são as principais responsáveis pelos altos estoques de carbono na floresta amazônica (Sist et al. 2014), cerca de 70\% nas áreas desse estudo. As árvores também são responsáveis pela formação dos estoques de carbono da necromassa (Chambers et al. 2000; Nascimento e Laurance 2002), e contribuem com um maior estoque de carbono abaixo do solo, devido à produção de raízes e a formação de agregados estáveis com material vegetal proveniente da necromassa (Chambers et al. 2000).

A abertura do dossel provocada pela supressão das árvores aumenta a exposiçấo do solo às altas temperaturas e à radiaçáo, ocasionando a oxidação da matéria orgânica do solo (Bedison et al. 2013) e, conseqüentemente, diminuição no estoque de carbono do solo. Além disso, a maior entrada de luz nos ambientes degradados promove mudanças na estrutura da vegetação, beneficiando herbáceas e arbustos. A supressão das árvores altera a estrutura florestal e, portanto, a dinâmica de retroalimentação entre os diferentes estoques de carbono.

Maior a degradação, maior o carbono do componente herbáceo e menor o carbono do solo e raízes. Esses resultados confirmam os adquiridos em ambiente sub-tropical (Giese et al. 2003; Rheinhardt et al. 2012) e indicam a incapacidade do componente herbáceo em manter os estoques de carbono no solo, em particular o componente raízes que poderia proporcionar uma proteção ao solo na ausência de cobertura florestal. Apesar de resultados mostrando que uma cobertura de gramíneas adequada pode conservar ou aumentar o estoque de carbono do solo através de uma densa rede de raízes (Salimon et al. 2009), os dados disponíveis para o ambiente ripário mostram o oposto. Esses resultados devem ser aprofundados porque a recente revisão do Código Florestal (Lei no 12.651 de 2012), que anistia o desmatamento em Áreas de Preservação Permanente (APP) antes de 2008 e reduz a necessidade de restaurar a mata ciliar a uma faixa de apenas 5 m para os imóveis rurais até quatro módulos fiscais, não levou em consideraçáo os efeitos de uma cobertura dominada por herbáceas no ambiente ripário.

Nas florestas ripárias conservadas, o estoque de carbono do solo é o segundo em importância (cerca de $30 \mathrm{Mg}(\mathrm{C})$ $\mathrm{ha}^{-1}$ nas áreas estudadas) e pode ser maior do que os estoques encontrados em florestas de terra firme (Filho-Novaes et al. 2007; Salimon et al. 2009). As altas taxas de umidade e a reduçấo da disponibilidade do oxigênio nos solos ripários ocasionam uma redução da decomposição de materiais vegetais, o que eleva os estoques de carbono (Moyano et al. 2013). As estimativas de carbono do solo desse estudo são conservadoras, pois não inclui as raízes grossas e nem as camadas mais profundas do solo. Salimon et al. (2009) reportam estoques de carbono orgânico até $100 \mathrm{~cm}$ de três a quatro vezes maiores que na profundidade 0-20 cm na Amazônia Oriental. Não há informação disponível se o perfil de acúmulo de $\mathrm{C}$ nos solos ripários é o mesmo do que na terra firme.

A reduçáo do estoque total de carbono da floresta com o processo de degradaçáo é esperada. Mas, esse estudo também evidencia a alta variabilidade nos estoques de carbono entre duas florestas ripárias próximas. Essa variabilidade é considerada normal em ambientes com alta heterogeneidade como a floresta amazônica (Nascimento e Laurance 2002) e, por isso, deve-se ter cuidado ao elaborar índices de estoque de carbono para a região. Avaliaçóes de campo, como neste estudo, fornecem subsídios para uma maior precisão na formulação de modelos de vegetação e carbono na Amazônia (Silva et al. 2013), assim como na valoração de serviços ambientais, a exemplo de projetos de Redução de Emissóes por Desmatamento e Degradação Florestal (REDD) (Besten e Verkooijen 2014). Este trabalho identificou uma forte relação entre o estoque de carbono dos diferentes compartimentos estudados nas florestas ripárias (biomassa viva acima do solo, necromassa, solo e total), principalmente a relação entre a biomassa viva acima do solo e o estoque total. Esse compartimento, em especial as árvores (DAP $>10 \mathrm{~cm}$ ), pode ser considerado como um proxy do estoque total para avaliaçôes de campo mais céleres.

O desmatamento das florestas ripárias leva a prejuízos socioeconômicos e ambientais enormes. Mesmo sendo protegidas por lei há cinco décadas, essas áreas sofrem com descaso e um intenso processo de degradação. $\mathrm{O}$ contexto socioeconômico da degradação da área de estudo caracterizado por Celentano et al. (2014) ressalta que a principal causa é a agricultura de corte-queima para a subsistência. As árvores 
grandes são retiradas primeiro para aproveitar a madeira ou fazer carvão, a vegetação remanescente é cortada e queimada para os plantios agrícolas. Nesse contexto, a aplicação da lei afeta direitamente a segurança alimentar de populaçóes carentes o que dificulta ainda mais a proteção dessas florestas. Com base nos resultados apresentados, o poder público poderia implementar a lei progressivamente com prioridade no combate a retirada das árvores grandes, mais fácil de implementar e mais eficiente para proteger os serviços ecológicos prestados pela floresta e a regeneração natural. Isso deve ser acompanhado de apoio técnico para orientar os agricultores a manejar melhor o fogo (com aceiros, cuidados com o vento, brigadas de incêndio, etc), efetuar uma transição para a agricultura ecológica (como roça sem fogo e sistemas agroflorestais) e restaurar as áreas degradadas. Conhecer a dinâmica dos diferentes compartimentos de carbono em um gradiente de degradaçáo fornece orientaçóes para restaurar e monitorar a restauraçáo, seja natural ou assistida.

\section{CONCLUSÃO}

As florestas ripárias conservadas armazenam grandes quantidades de carbono, principalmente devido às árvores de grande porte. $\mathrm{O}$ estoque de carbono nos diferentes compartimentos (biomassa viva acima do solo, necromassa e solo) estão fortemente relacionados entre si e também com a abertura do dossel. A degradação leva a redução expressiva de carbono em todos esses compartimentos. Nas áreas conservadas, a biomassa viva acima do solo tem a maior participação no estoque total de carbono, mas com a degradação da floresta, o solo vem a ser o compartimento com maior participaçáo no estoque total apesar de sua reduçáo drástica pela degradação. O estoque de carbono nas árvores de grande porte pode servir de proxy não somente para o estoque total de carbono mas também para outros serviços ecológicos essenciais providos pelas florestas ripárias. A incorporação desses resultados em modelos regionais de carbono pode auxiliar na implementação e revisão do Código Florestal Brasileiro, em particular na restauração das florestas ripárias onde a agricultura está consolidada.

\section{AGRADECIMENTOS}

Este projeto foi financiado pela Fundação de Amparo à Pesquisa e ao Desenvolvimento Científico e Tecnológico do Maranhão (FAPEMA) e pela Coordenação de Aperfeiçoamento de Pessoal de Nível Superior (CAPES). Agradecemos a todos os colaboradores do trabalho de campo nas comunidades Pepital e Só Assim em Alcântara, em especial ao agricultor João Maleta, e aos estudantes do curso de Agronomia da Universidade Estadual do Maranhão que apoiaram no campo e no laboratório. Marilda Mascarenhas, Cláudio Farias e a Oficina de Comunicação e Arte (OCA) deram apoio logístico essencial a realização do projeto. Somos gratos a três revisores anônimos pelas diversas correçóes e sugestôes que melhoraram muito a qualidade do manuscrito.

\section{BIBLIOGRAFIA CITADA}

Arevalo, A. 2002. Metodologia para estimar o estoque de carbono em diferentes usos da terra. Embrapa Floresta, Colombo, 2002, 73p.

Bedison, J.E.; Scatena, F.N.; Mead, J.V. 2013. Influences on the spatial pattern of soil carbon and nitrogen in forested and non-forested riparian zones in the Atlantic Coastal Plain of the Delaware River Basin. Forest Ecology and Management, 302: 200-209.

Besten, J. W.; Verkooijen, P. 2014. The evolution of REDD+: An analysis of discursive-institutional dynamics. Environmental Science and Policy, 35: 40-48.

Brito, C.M.S.; Rego, M.M.C. 2001. Community of male Euglossini bees (Hymenoptera: Apidae) in a secondary forest, Alcântara, MA, Brazil. Brazilian Journal Biology, 61: 631-638.

Brown, S. 2002. Measuring, monitoring, and verification of carbon benefits for forest-based projects. Philosophical Transactions Royal Society, 360: 1669-1684.

Bueno, A. S.; Bruno, R. S.; Pimentel, T. P.; Sanaiotti, T. M.; Magnusson, W. E. 2012. The width of riparian habitats for understory birds in an Amazonian Forest. Ecological Applications, 22: 722-734.

Celentano, D.; Rousseau, G.X.; Engel, V.L.; Façanha, C.L.; Oliveira, E.M.; Moura, E.G. 2014. Perceptions of environmental change and use of tradicional knowledge to plan riparian Forest restoration with relocated communities in Alcantara, Eastern Amazon. Journal of Ethnobiology and Ethnomedicine, 10: 1-14.

Chambers, J.Q.; Higuchi, N.; Schimel, J.P.; Ferreira, L.V.; Melack, J.M. 2000. Decomposition and carbon cycling of dead trees in tropical forests of the central Amazon. Oecologia, 122: 380-388.

Chave, J.; Andalo, C.; Brown, S.; Cairns, M.A.; Chambers, J.Q.; Eamus, D.; et al. 2005. Tree allometry and improved estimation of carbon stocks and balance in tropical forests. Oecologia, 145: 87-99.

Di Rienzo J.A.; Casanoves F.; Balzarini M.G.; Gonzalez L.; Tablada M.; Robledo C.W. 2011. InfoStat versión 2011. Grupo InfoStat, FCA, Universidad Nacional de Córdoba, Argentina. (http:// www.infostat.com.ar). Acesso em 05/04/2013.

FAO. 2009. Towards defining forest degradation: Comparative analysis of existing definitions. Forest Resources Assessment Programme Working Paper 154. FAO, Roma, 2009, 62p.

Filho-Novaes, J.P.; Selva, E.C.; Couto, E.G.; Lehmann, J.; Johnson, M.S.; Riha, S.J. 2007. Distribuição espacial de carbono em solo sob floresta primária na Amazônia meridional. Revista Árvore, 31: 83-92.

Frangi, J.L.; Lugo, A.E. 1985. Ecosystem dynamics of a subtropical floodplain forest. Ecological Monographs, 55: 351-369.

Gehring, C.; Park, S.; Denich, M. 2004. Liana allometric biomass equation for Amazonian primary and secondary forest. Forest Ecology and Management, 195: 69-83. 
Gehring, C.; Park, S.; Denich, M. 2008. Close relationship between diameters at $30 \mathrm{~cm}$ height and at breast height (DBH). Acta Amazonica, 38: 65-70.

Gehring, C.; Zelarayan M.L.C.; Almeida, R. 2011. Allometry of the babassu palm growing on a slash-and-burn agroecosystem of the eastern periphery of Amazonia. Acta Amazonica, 41: 127-134.

Giese, L.A.B.; Aust, W.M.; Kolka, R.K.; Trettin, C.C. 2003. Biomass and carbon pools of disturbed riparian forests. Forest Ecology and Management, 180: 493-508.

Hazlett, P.W.; Gordon, A.M.; Sibley, P.K.; Buttle, J.M. 2005. Stand carbon stocks and soil carbon and nitrogen storage for riparian and upland forests of boreal lakes in northeastern Ontario. Forest Ecology and Management, 219: 56-68.

Houghton, R.A.; Skole, D.L.; Nobre, C.A.; Hackler, J.L.; Lawrence, K.T.; Chomentowski, W.H. 2000. Annual fluxes of carbon from deforestation and regrowth in the Brazilian Amazon. Nature, 403: 301-304.

INPE. Instituto Nacional de Pesquisas Espaciais. 2014. Monitoramento da Floresta Amazônica Brasileira por SatéliteProjeto Prodes. (http://www.obt.inpe.br/prodes/). Acesso em 12/06/2014.

IPCC. 2007. Fourth Assessment Report on climate change impacts, adaptation and vulnerability of the Intergovernmental Panel on Climate Change. Cambridge University, Cambridge, 2007, 939p.

IPCC. 2014. Climate Change 2014: Impact, Adaptation and Vulnerability. Assessment Report of the Intergovernmental Panel on Climate Change. Cambridge University, Cambridge, 1820p.

Jones, E.B.D.; Helfman, G.S.J.; Harper O; Bolstad, P.V. 1999. Effects of riparian forest removal on fish assemblages in southern Appalachian streams. Conservation Biology, 13: 1454-1465.

Junk, W.J.; Ohly, J.J.; Piedade, M.T.F.; Soares, M.G.M. 2000. The Central Amazon floodplain: Actual use and options for a sustainable management. Backhuys Publishers, Leiden, 2000, 590 p.

Kumar, M.; Nair, R. 2011. Carbon sequestration Potential of Agroforestry Systems, Opportunities and Challenges. Advances in Agroforestry, Springer, Nova Iorque, 2011, 307p.

Lees, A.C.; Peres C.A. 2008. Conservation value of remnant riparian forest corridors of varying quality for Amazonian birds and mammals. Conservation Biology, 22: 439-449.

Malhi, Y.; Wood, D.; Baker, T.R.; Wright, J.; Phillips, O.L.; Cochrane, T.; et al. 2006. The regional variation of aboveground live biomass in old-growth Amazonian forests. Global Change Biology, 12: 1.107-1.138.

Malhi, Y.; Timmons R.J.; Betts, R.A.; Killeen, T.J.; Li, W.; Nobre, C.A. 2008. Climate change, deforestation and the fate of the Amazon. Science, 319: 169-172.

MCT. 2013. Estimativas anuais de emissöes de gases de efeito estufa no Brasil. Ministério da Ciência, Tecnologia e Inovação, Brasília, 2013, 80p.

Moyano, E. F.; Manzoni, S.; Chenu, C. 2013. Responses of soil heterotrophic respiration to moisture availability: An exploration of processes and models. Soil Biology and Biochemistry, 59: 72-85.
Nascimento, H.E.M.; Laurance, W.F. 2002. Total aboveground biomass in central Amazonian rainforests: a landscape-scale study. Forest Ecology and Management, 68: 311-321.

Nelson, B.W.; Mesquita, R.; Pereira, J.L.G.; de Souza, S.G.A.; Batista, G.T.; Couto, L.B. 1999. Allometric regressions for improved estimate of secondary forest biomass in the central Amazon. Forest Ecology and Management, 117: 149-167.

Newbold, J.D.; Susan H.B.W.; Sweeney, P.K.; Stephen, J.A. 2010. Water quality functions of a 15-Year-Old riparian forest buffer system. Journal of the American Water Resources Association, 46: 299-310.

Nogueira, E.M.; Fearnside, P.M.; Nelson, B.W. 2008. Normalization of wood density in biomass estimates of Amazon forests. Forest Ecology and Management, 256: 990-996.

Pearson, T.R.H.; Brown, S.L.; Birdsey, R.A. 2007. Measurement guidelines for the sequestration of forest carbon. US Forest Service, Newtown Square, 2007, 42 p.

Rheinhardt, R.; Brinson, M.; Meyer, G.; Miller, K. 2012. Integrating forest biomass and distance from channel to develop an indicator of riparian condition. Ecological Indicators, 23: 46-55.

Rieger, I.L.; Friederike, K.I.; Cierjacks, A. 2014. The interplay of sedimentation and carbon accretion in riparian forests. Geomorphology, 24: 157-167.

Salimon, C.I.; Wadt, P.G.S.; Alves, S.S. 2009. Decrease in carbon stocks in an oxisol due to land use and cover change in the Southwestern Amazon. Ambiente e Água, 4: 57-65.

Silva, F.B.; Shimabukuro, Y.E.; Aragao, L.E.O.C.; Anderson, L.O.; Pereira, G.; Cardozo, F.; Arai, E. 2013. Large-scale heterogeneity of Amazonian phenology revealed from 26-year long AVHRR/ NDVI time-series. Environment Research Letters, 8: 1-12.

Sist P.; Mazzei L.; Blanc L.; Rutishauser E. 2014. Large trees as key elements of carbon storage and dynamics after selective logging in the Eastern Amazon. Forest Ecology and Management, 318: 103-109.

Van Wagner, C.E. 1968. The line intersect method in forest fuel sampling. Forest Science, 14: 20-26.

Walkley, A.; Black, I. 1934. An examination of the Degtjareff method and a proposed modification of the chromic matter and a proposed modification of the chromic acid titration method. Soil Science, 34: 29-38.

Xavier, G.P.S. 2009. Mudanças no estoque de carbono da biomassa lenhosa de florestas de várzea baixa da Amazônia central ao longo de um gradiente sucessional. Instituto Nacional de Pesquisas Amazônicas, Manaus, 2009, 61p.

Zanne, A.E.; Lopez-Gonzalez, G.; Coomes, D.A.; Ilic, J.; Jansen, S.; Lewis, S.L.; Miller, R.B.; Swenson, N.G.; Wiemann, M.C.; e Chave, J. 2009. Global wood density database (http://www. worldagroforest- ry.org/sea/Products/AFDbases/WD). Acesso em 12/06/2014

Recebido em 09/02/2015

Aceito em 23/02/2015 
